Cahiers de philosophie de l'université de

\title{
Callistique naturaliste et théorie humaniste de la peinture
}

\section{Carole Talon-Hugon}

\section{(2) OpenEdition}

1 Journals

Édition électronique

URL : https://journals.openedition.org/cpuc/623

DOI : $10.4000 /$ cpuc.623

ISSN : 2677-6529

Éditeur

Presses universitaires de Caen

\section{Édition imprimée}

Date de publication : 31 décembre 2014

Pagination : 41-54

ISBN : 978-2-84133-512-1

ISSN : 1282-6545

\section{Référence électronique}

Carole Talon-Hugon, «Callistique naturaliste et théorie humaniste de la peinture », Cahiers de philosophie de l'université de Caen [En ligne], 51 | 2014, mis en ligne le 13 juin 2018, consulté le 01 février 2023. URL : http://journals.openedition.org/cpuc/623 ; DOI : https://doi.org/10.4000/cpuc.623

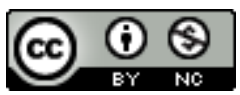

Creative Commons - Attribution - Pas d'Utilisation Commerciale 4.0 International - CC BY-NC 4.0 https://creativecommons.org/licenses/by-nc/4.0/ 


\section{Callistique naturaliste et théorie humaniste de la peinture}

$\mathrm{E}$ N INVENTANT LA CATÉGORIE DES «BEAUX-ARTS " ${ }^{1}$, le XVIII ${ }^{\mathrm{e}}$ siècle bouleversa et contribua à ruiner les catégorisations anciennes d'arts mécaniques et d'arts libéraux, reconfigura le champ des activités humaines et attribua à un certain nombre d'entre elles une finalité avant tout esthétique, que soulignait ce nouveau nom. Or, faire de la beauté le caractère distinctif et la valeur suréminente d'un certain type d'activités, c'était enrôler ces arts dans le vaste mouvement de subjectivisation qui affecte le beau au début des temps modernes. L'histoire des arts et plus encore celle de l'idée d'art rencontra ainsi une autre évolution largement indépendante de la première : celle de l'idée de beauté. Cette rencontre produisit un certain nombre de turbulences, particulièrement visibles dans l'œuvre de Diderot. Elles prennent chez ce dernier la forme d'une tension entre ses réflexions sur le beau d'une part, principalement exposées dans ses Recherches philosophiques sur l'origine et la nature du beau, et ses écrits sur l'art d'autre part ${ }^{2}$. Alors que les premières fondent une callistique naturaliste, les seconds relèvent d'une conception humaniste de l'art.

\section{Une callistique naturaliste}

Pour mesurer toute la modernité des réflexions de Diderot sur la beauté, il faut rappeler les traits les plus saillants de la conception pré-moderne du beau.

1. Même si le périmètre des nouveaux beaux-arts est variable, l'appellation s'impose de manière progressive en Europe. Du Bos l'utilise dans ses Réflexions critiques sur la poésie et la peinture, en 1719, Voltaire dans son Temple du goût en 1733, Batteux dans Les Beaux-arts réduits à un même principe en 1746, Lacombe dans son Dictionnaire portatif des beaux-arts en 1752, Sulzer dans sa Théorie générale des beaux-arts en 1771, etc.

2. On se bornera ici à ceux qui portent sur la peinture. 
L'Antiquité ou le Moyen Âge ne concevaient pas la beauté sensible comme une réalité autonome et indépendante d'une transcendance. Pour Platon, ou pour Plotin, elle n'existe que par participation à l'Idée intelligible de beauté, soit à la Beauté absolue, éternelle et immuable. Le Moyen Âge chrétien fit de la beauté une propriété de l'Être suprême qu'est Dieu et y vit une beauté supra-substantielle qui rend belles les choses sensibles qui proviennent de Lui. La beauté était donc indissociablement splendeur sensible et métaphysique. Cette origine transcendante du beau le liait avec le bien et avec le vrai, comme on le voit dans la kaloskagatia des Grecs ou dans la convertibilité des transcendantaux au Moyen Âge. Ces valeurs ne font qu'un dans l'Être; elles sont des prédicaments de la divinité. Elles ne diffèrent que lorsqu'on se place du point de vue du sujet humain: pour lui, le bien c'est l'Être dans sa dimension conative, le beau c'est l'Être dans sa dimension perceptive ${ }^{3}$. Ainsi, à la question de savoir "si ces objets sont beaux parce qu'ils plaisent, ou s'ils plaisent parce qu'ils sont beaux " ${ }^{4}$, la pensée pré-moderne du beau soutient avec saint Augustin : «ils plaisent parce qu'ils sont beaux " 5 . Comme les choses bonnes, les choses belles sont ce qu'elles sont indépendamment des regards et des jugements humains ${ }^{6}$. Le beau est donc objectif et ontologiquement autosubsistant.

Bien des passages de l'œuvre de Diderot semblent renvoyer à une telle métaphysique du beau. L'enthousiasme qu'il éprouve à la lecture de l'Essai sur le mérite et la vertu de Shaftesbury paraît aller dans ce sens ${ }^{7}$. Shaftesbury ne déclarait-il pas en effet dans Characteristics of Men, Manners, Opinions, Times, à la suite des platoniciens de Cambridge, que le beau et le bien ne sont qu'un: "ce qui est beau est harmonieux et proportionné: ce qui est harmonieux et proportionné est vrai; et ce qui est du même coup et beau et vrai est, par conséquent, agréable et bon ${ }^{8}$ ? Ne soutenait-il pas, comme Diotime dans Le Banquet de Platon, que l'initiation au beau se fait par

3. Saint Thomas d'Aquin, Somme théologique, question 5, art. 5, sol. 1.

4. Saint Augustin, De vera religione, chap. 32, in Euvres, J. Clémence et J. Pegon (trad. fr.), Paris, Desclée de Brouwer (Bibliothèque augustinienne), vol. VIII, 1951, p. 111.

5. Ibid.

6. «La vertu possède par elle-même un certain éclat grâce auquel elle est belle, même si elle n'était reconnue par personne", affirme Albert le Grand, De pulchro et bono, partie commentaire sur les noms divins, cité par U. Eco dans Art et Beauté dans l'esthétique médiévale, Paris, B. Grasset, 1997, p. 50 (nous soulignons).

7. «Je l'ai lu et relu; je me suis rempli de son esprit; et j'ai, pour ainsi dire fermé son livre, lorsque j'ai pris la plume», écrit Diderot dans son «Discours préliminaire» à l'Essai de $M$. $S^{* * *}$ sur le mérite et la vertu, in Euvres complètes, H. Dieckmann et al. (éd.), Paris, Hermann, t. I, Le Modèle anglais, 1975, p. 300.

8. Shaftesbury, Characteristics of Men, Manners, Opinions, Times, Hildesheim - New York, G. Olms, 1978, t. III, p. 183 [fac-similé de l'édition de Londres, 1711]. 
degrés, depuis la beauté des «formes mixtes » ${ }^{9}$ établies par l'homme ou par la nature, en passant par les «formes qui forment» ${ }^{10}$, celles en lesquelles se trouvent et l'esprit et l'effet de l'esprit, jusqu'au "principe, source et fontaine de toute beauté ${ }^{11}$ qui est la beauté même par laquelle les esprits sont beaux? Or, Diderot dit épouser pleinement ces propos. Il n'est certes alors qu'au début de sa carrière philosophique, et on pourrait voir là un enthousiasme juvénile et provisoire. Mais beaucoup de textes plus tardifs semblent aller dans le même sens, celui d'une croyance dans la convertibilité des transcendantaux. Ainsi écrit-il en 1765 dans ses Essais sur la peinture, «[1] e vrai, le bon et le beau se tiennent de bien près. Ajoutez à l'une des deux premières qualités quelque circonstance rare, éclatante, et le vrai sera beau, et le bon sera beau ${ }^{12}$. En 1775, il déclare encore dans une formule d'allure très plotinienne, que «[le] beau [...] n'est jamais que l'éclat du bon ${ }^{13}$. Le goût n'est-il pas, d'ailleurs, défini comme "une facilité [...] à saisir le vrai ou le bon avec la circonstance qui le rend beau " ${ }^{14}$ ? Le Neveu de Rameau ne pose-t-il pas entre ces qualités un lien d'engendrement: "le vrai qui est le Père, et qui engendre le bon qui est le Fils, d'où procède le beau qui est le Saint-Esprit ${ }^{15}$ ?

Pourtant, beaucoup de choses éloignent Diderot d'un tel spiritualisme; car s'il a été enthousiasmé par Shaftesbury, il a été convaincu par Hutcheson et par sa Recherche sur l'origine de nos idées de la beauté et de la vertu. Cet ouvrage, publié en 1725, a tiré les conséquences pour la question du beau de la révolution scientifique de l'âge classique. La nouvelle epistémè qui s'est progressivement imposée conçoit le sensible d'une manière radicalement neuve. Pour tous ceux qui font leur la nouvelle physique mécaniste corpusculaire, le monde sensible, fait d'objets étendus, colorés, pesants, sonores, odorants, etc., n'existe en tant que tel que pour un sujet. Le bleu, l'aigu, l'acide, le soyeux ou le rugueux n'existent pas tels quels dans l'objet; ils ne sont rien hors de la sensation. De cette subjectivisation du sensible s'ensuivent des conséquences considérables pour la question de la beauté, conséquences d'abord inaperçues, mais qui se font clairement sentir au XVIII ${ }^{\mathrm{e}}$ siècle.

9. Ibid., t. II, p. 406 .

10. Ibid., p. 407.

11. Ibid.

12. D. Diderot, Essais sur la peinture, in Essais sur la peinture - Salons de 1759, 1761, 1763, G. May et J. Chouillet (éd.), Paris, Hermann, 2007, p. 76.

13. D. Diderot, Plan d'une université pour le gouvernement de Russie, in Euvres complètes de Diderot, J. Assézat et M. Tourneux (éd.), Paris, Garnier, t. III, 1875, p. 478.

14. D. Diderot, Essais sur la peinture, p. 78.

15. D. Diderot, Le Neveu de Rameau, in Euvres complètes, t. XII, Le Neveu de Rameau, 1989, p. 462. 
Celles-ci sont tout entières contenues dans cette phrase de la Recherche sur l'origine de nos idées de la beauté et de la vertu: " on voudra bien noter que dans la suite de cet ouvrage, le mot beauté est pris pour l'idée qu'elle suscite en nous» ${ }^{16}$. Que dit-elle en effet? Que le beau n'est pas une essence, qu'il n'a pas de consistance ontologique propre. À tel point que «s'il n'existait aucun esprit possédant un sens de la beauté pour contempler ces objets [que nous disons beaux] », affirme Hutcheson, «je ne vois pas comment on pourrait les dire beaux ${ }^{17}$. La subjectivisation du beau signifie aussi, par voie de conséquence, la fin de la convertibilité des transcendantaux: en refusant aux beautés sensibles toute attache transcendante, le beau s'autonomise du bien comme du vrai. Tel est ce phénomène d'esthétisation du beau produit par la vision subjectiviste, psychologisante et sensualiste des modernes: le beau n'est plus que beau et n'existe que dans une expérience sensible.

Dans le sillon ouvert par cet ouvrage, Diderot publie, quatorze ans plus tard, ses Recherches philosophiques sur l'origine et la nature du beau (ouvrage désormais noté Recherches). En y affirmant que le beau n'est que la «qualité» de ce qui est dit beau ${ }^{18}$, Diderot exclut toute forme de réalisme transcendant. Le beau n'est pas, comme le soutenaient Platon ou Plotin, une essence supraempirique. Il n'existe pas de beau en soi, mais seulement des choses belles. C'est là une première réduction décisive: Diderot prend le parti d'Hippias contre Socrate dans l'Hippias majeur: la beauté n'existe pas; il n'y a que des objets beaux. Toutefois, si soutenir que le beau n'est que la «qualité» de ce qui est dit beau exclut un réalisme essentialiste, cela n'interdit pas pour autant de penser un réalisme des propriétés, autrement dit de soutenir que les propriétés esthétiques sont possédées par les choses auxquelles on les attribue; qu'elles en sont des caractéristiques réelles. Est-ce le cas de Diderot? Non : il exclut cette possibilité comme la précédente. Car il partage avec Hutcheson l'affirmation de la subjectivité de l'idée de beauté. La beauté n'est pas une Idée au sens platonicien du terme, mais une idée au sens moderne, c'est-à-dire un contenu de conscience et rien de plus qu'un contenu de conscience. Le beau est une idée; non pas innée, non pas donnée à l'homme par la Révélation, mais issue - comme toutes les autres idées - de l'expérience. Diderot est donc antiréaliste au sens où il considère que ces propriétés ne sont pas des caractéristiques réelles des objets du monde, mais qu'elles sont subjectives, c'est-à-dire corrélatives à certains états mentaux.

16. F. Hutcheson, Recherche sur l'origine de nos idées de la beauté et de la vertu [1725], I, I, IX, A.-D. Balmès (trad. fr.), Paris, J. Vrin, 1991, p. 55.

17. Ibid., I, I, XVI, p. 60.

18. D. Diderot, Recherches philosophiques sur l'origine et la nature du beau, in Euvres esthétiques, P. Vernière (éd.), Paris, Garnier, 1994, p. 417 (cette édition sera désormais notée Recherches). 
Mais qu'entend Diderot exactement par là ? Car c'est en plusieurs sens qu'on peut soutenir que le beau est une propriété subjective. On peut notamment vouloir dire que cette propriété n'est que la projection de quelque chose du sujet sur l'objet. Par exemple, que c'est le plaisir qu'il nous procure qui nous le fait trouver beau, et non sa beauté qui nous procure du plaisir. Dans une telle conception esthétique, le beau est la projection de nos affects sur les objets du monde et la croyance que ce sont les traits objectifs de ces objets qui suscitent nos affects passe pour une illusion. Est-ce là la position de Diderot? Non. Dans ses Recherches, il apporte à la question «cela est-il beau parce qu'il plaît? Ou cela plaît-il parce qu'il est beau ? ${ }^{19}$, une réponse sans équivoque: après avoir rappelé que saint Augustin répondrait par la seconde proposition, il ajoute: «Je le crois comme [lui] ${ }^{20}$. Quelques pages plus loin, c'est d'ailleurs pour la même raison qu'il réfute Wolf: il «semble prétendre [...] qu'une chose est belle parce qu'elle nous plaît; au lieu qu'elle nous plaît parce qu'elle est belle, comme Platon et saint Augustin l'ont très bien remarqué » ${ }^{21}$. Ainsi, le beau est bien, pour Diderot, un état mental subjectif, mais cet état mental subjectif n'est pas arbitrairement lié à tel ou tel objet.

Il résulte de ce qu'on vient de voir que Diderot n'est ni réaliste, ni radicalement subjectiviste. Il y aurait là une incohérence si ces deux options étaient les seules possibles. Mais ce n'est pas le cas. La position de Diderot s'éclaire et devient tout à fait cohérente sur ce point lorsqu'on utilise pour la penser la catégorie de propriété dispositionnelle, qui désigne des propriétés ni strictement subjectives ni strictement objectives: des propriétés qui naissent du contact de l'esprit humain et des choses. On notera effectivement que Diderot, dans l'énumération d'adjectifs choisis pour qualifier le beau, n'utilise pas celui d' «absolu». Et en effet, absolu signifierait sans lien et désignerait une propriété autosubsistante, indépendante de toute appréhension humaine. En revanche, il emploie les adjectifs «réel », « essentiel » et «vrai ». «Réel» renvoie à la chose, « essentiel » à la nature de la chose, et «vrai » à son objectivité; aucun de ces mots ne fait signe vers une quelconque forme d'irrelativité. La beauté n'est pas une qualité objective des choses comme l'est leur masse par exemple, mais elle n'est pas non plus une simple appréciation idiosyncrasique; elle est bien une qualité-de-l'objet-pour-un-sujet-humain. Comme la douceur n'est pas dans le fruit mais n'est pas sans le fruit, la qualité dispositionnelle n'est pas dans la chose mais dans le lien qui unit certaines configurations dans

\footnotetext{
19. Ibid., p. 393 .

20. Ibid.

21. Ibid., p. 394 .
} 
la chose et le sujet qui les perçoit. Les qualités esthétiques sont relatives, mais relatives à l'homme en général. Diderot ne soutient donc ni un réalisme radical, ni un subjectivisme radical; parce que «subjectif» ne signifie pas «individuel», il y a place dans son texte pour une forme de réalisme modéré.

Exposer la nature de l'idée de beauté n'est pas encore dire ce que doit être une chose pour faire surgir cette idée en nous. Qu'en est-il donc précisément de cette configuration dans les choses, qui nous les font trouver belles ou bonnes? Pour répondre à cette question, il faut remonter de l'épreuve de la beauté aux propriétés qui, dans l'objet, l'occasionnent. Cette démarche inductive de Diderot était déjà celle d'Hutcheson: «nous tâcherons de découvrir quelle est l'occasion immédiate de ces idées agréables, ou quelle est dans les objets la qualité réelle qui les excite habituellement», déclarait-il au début de son traité ${ }^{22}$. C'est dans l'«uniformité dans la diversité» qu'il trouve cette "occasion immédiate», cette "qualité réelle» ordinairement inaperçue: «les figures qui excitent en nous les idées de beauté semblent être celles dans lesquelles il y a de l'uniformité au sein de la variété ${ }^{23}$.

De la même manière, Diderot cherche à savoir ce que sont les propriétés dans l'objet qui provoquent en l'homme l'idée de beauté. Il ne suffit pas d'invoquer la "belle nature» comme Batteux s'est, à tort, contenté de le faire; il faut encore la "définir», écrit-il dans sa Lettre sur les sourds et muets ${ }^{24}$. Or, qu'est-ce que la définir sinon exposer sa survenance? Dans ses Recherches, Diderot passe en revue les qualités dans lesquelles les auteurs ont fait consister le beau phénoménal: saint Augustin soutient que l'essence du beau est l'unité; Crouzat fixe ses caractères dans la variété, l'unité, la régularité, l'ordre et la proportion; Shaftesbury fait du beau l'utile; le père André le fait consister dans la proportion, l'ordre et la symétrie. C'est la position de ce dernier que retient Diderot.

En 1745, il affirmait déjà, en marge de sa traduction de Shaftesbury, que la beauté cesse où cessent l'harmonie, l'ordre et la proportion. Ces principes sont maintes fois repris dans ses Salons. En 1751, les Recherches le redisent clairement: «il n'entre dans la notion de beau [...] que les notions d'ordre, de rapports, de proportion, d'arrangement, de symétrie, de convenance, de disconvenance ${ }^{25}$. En désignant les caractéristiques formelles du beau phénoménal par les termes de proportion, d’ordre et de symétrie, Diderot

22. F. Hutcheson, Recherche sur l'origine de nos idées..., I, I, IX, p. 55.

23. Ibid., I, II, III, p. 62.

24. D. Diderot, Lettre sur les sourds et muets, in Euvres complètes, t. IV, Le Nouveau Socrate - Idées II, 1978, p. 182.

25. D. Diderot, Recherches, p. 417. 
retrouve la veine de la tradition pythagoricienne. Mais là encore, il n'y a aucune place pour un quelconque réalisme des idées: ces idées sont des idées factices, issues de l'exercice de la sensorialité; elles ne sont que des "abstractions de notre esprit ${ }^{26}$. À ces catégories d'ordre, de proportion et de symétrie, Diderot ajoute un terme qui tend à les supplanter et à devenir un hyperonyme: celui de « rapport». À maintes reprises, Diderot subsume sous ce mot les catégories descriptivo-évaluatives issues de la tradition; c'était déjà le cas en 1748 dans le premier des Mémoires sur différents sujets de mathématiques, ou encore en 1751 dans une lettre à $M^{\text {lle }}$ de la Chaux explicitant la Lettre sur les sourds et muets. Le lien qui unit l'idée de rapport à celles de proportion ou de symétrie est clair : la proportion est un rapport de grandeur entre les parties d'une chose ou entre la chose et l'une de ses parties; la symétrie, un rapport de similitude entre des parties; l'harmonie, un rapport qu'entretiennent les parties d'un tout pour former un ensemble agréable, etc. Aussi Diderot peut-il écrire: «j'appelle donc beau hors de moi, tout ce qui contient en soi de quoi réveiller dans mon entendement l'idée de rapport; et beau par rapport à moi, tout ce qui réveille cette idée ${ }^{27}$. Cette idée de rapport permet en outre d'expliquer les degrés de beauté: ils sont fonction de la capacité des objets à «réveill[er] en moi le plus d'idées de rapports, et le plus de certains rapports ${ }^{28}$.

La beauté doit donc être pensée en termes de survenance. Survenir, c'est être en relation avec ces propriétés, varier en fonction de leur variation, mais non pas se réduire à elles. Cette idée de covariation est bien exprimée par Diderot lorsqu'il parle, dans ce même texte, de ces qualités dans l'objet «par [lesquelles] la beauté commence, augmente, varie à l'infini, décline et disparaît " ${ }^{29}$. La beauté survient donc de ces propriétés: "Les figures, les proportions, les mouvements et les couleurs de ceux-ci ne sont pas plutôt exposés à nos yeux, qu'il résulte de l'arrangement et de l'économie de leurs parties une beauté qui nous récrée, ou une difformité qui nous choque ${ }^{30}$. Diderot adosse ainsi la qualité strictement évaluative "être beau ", à des propriétés descriptivo-évaluatives : «être proportionné », «être symétrique», etc. Il y a là un gain d'intelligibilité car ces dernières propriétés ont un degré d'objectivité que le beau n'a pas: elles sont, écrit Diderot, "positives", «distinctes», «nettes» et « réelles ${ }^{31}$.

\footnotetext{
26. Ibid., p. 416.

27. Ibid., p. 418.

28. Ibid., p. 420.

29. Ibid., p. 418.

30. D. Diderot, Essai de M. $S^{* * *}$ sur le mérite et la vertu, p. 321.

31. D. Diderot, Recherches, p 416.
} 
Leur objectivité est plus grande que celle de la qualité purement évaluative de beauté, mais toutefois moindre que celle des propriétés purement descriptives. Aussi Diderot poursuit-il l'espoir de remonter de celles-là à celles-ci, autrement dit de montrer que l'harmonie, la proportion et la symétrie, à leur tour, surviennent de propriétés qui, elles, sont strictement descriptives. C'est ce qu'on lit dans la suite de la phrase partiellement citée plus haut: «il n'entre dans la notion de beau [...] que les notions d'ordre, de rapports, de proportion, d'arrangement, de symétrie, de convenance, de disconvenance; ces notions ne découlant pas d'une autre source que celles d'existence, de nombre, de longueur, largeur, profondeur... dont on ne conteste point ${ }^{32}$. Les choses mesurables sont des propriétés parfaitement objectives et indiscutables ( on ne [les] conteste point»). Ainsi, le beau survient de qualités descriptivoévaluatives qui, à leur tour, surviennent de propriétés descriptives. Il repose in fine sur des traits non esthétiques renvoyant à des propriétés objectives mesurables et incontestables.

A-t-on atteint l'ultime étape de l'explication? Nous connaissons la nature du beau (une idée) et son origine (sa survenance). Mais reste une question décisive: pourquoi certaines dispositions des objets sensibles que nous désignons par les termes d'harmonie, de proportion ou de rapport nous plaisent-elles? Diderot reproche au père André, qui avait eu le mérite de définir la beauté par la proportion, l’ordre et la symétrie, de n'avoir pas "développ[é] l'origine des notions qui se trouvent en nous, de rapport, d'ordre, de symétrie ${ }^{33}$. Autrement dit, même en possession du concept de rapport, on n'a pas achevé la généalogie du beau. C'est la physiologie qui fournit à Diderot la réponse à cette ultime question. Considérons en effet les idées d'ordre, d'arrangement et de symétrie: elles ont, écrit Diderot, «leur origine dans nos besoins ${ }^{34}$; et les bonnes proportions sont celles qui permettent «l'accomplissement des fonctions animales» ${ }^{35}$. On voit ici tout ce qui sépare Diderot de la tradition pythagoricienne à laquelle il emprunte ces qualités esthétiques. Alors que l'horizon d'intelligibilité de celle-ci était cosmologique, il est pour Diderot, biologique. La racine du beau est finalement l'utile qui, ainsi qu'il l'écrit dans ses Pensées sur l'interprétation de la nature, "circonscrit tout». Le Salon de 1767 donne une expression plus développée de cette formule lapidaire: «La nature est bonne et belle quand elle nous favorise. Elle est laide et méchante quand elle nous afflige ${ }^{36}$.

32. D. Diderot, Recherches, p. 417 (nous soulignons).

33. Ibid., p. 415.

34. Ibid., p. 416.

35. D. Diderot, Essai de M. $S^{* * *}$ sur le mérite et la vertu, p. 324.

36. D. Diderot, Salons, III. Ruines et paysages. Salon de 1767, E. M. Bukdahl et al. (éd.), Paris, Hermann, 2008, p. 187. 
Ainsi donc, Diderot procède ultimement à une naturalisation du beau. La beauté n'est plus une essence, les choses sensibles ne reçoivent pas leur beauté d'une idée métaphysique ou de la splendeur divine; la beauté est une qualité des objets proportionnés de telle manière qu'ils favorisent la vie humaine.

\section{Une théorie humaniste des arts}

Avant le XVIII e siècle, les réflexions sur l'art ne relevaient pas d'une esthétique, et les réflexions sur la beauté ne concernaient que très partiellement les arts. Le substantif «esthétique» n'était pas encore en usage, et aucun champ disciplinaire constitué n'attendait sa dénomination. Les réflexions théoriques sur les arts ne relevaient pas d'une approche esthétique, mais s'inscrivaient dans des perspectives variées: politiques (pensons au livre $\mathrm{X}$ de La République de Platon), poḯtiques (pensons à La Poétique d'Aristote), ou encore théologiques (pensons à la querelle des images qui fit rage à Byzance au cours des VIII et IX ${ }^{\mathrm{e}}$ siècles). C'est que les fonctions assignées aux arts n'étaient pas qu'esthétiques, et même n'étaient pas principalement esthétiques. Elles étaient aussi magiques, thaumaturgiques, cultuelles, religieuses, éthiques ou politiques. L'artistique et l'esthétique ne se confondaient pas. Mais quand le beau devient valeur suréminente autour de laquelle se construit la catégorie nouvelle de beaux-arts, on peut penser que la mutation radicale dans la manière d'entendre le beau que nous venons de voir n'est pas sans effet sur ceuxci. Le cas de Diderot est particulièrement intéressant puisqu'il est non seulement théoricien du beau, mais aussi théoricien de l'art, écrivain et critique. Ses Recherches traitent de la beauté en général et non de l'art en particulier et défendent l'anthropologie du beau que nous venons d'analyser. Que retrouve-t-on de celle-ci dans ses Salons et ses autres écrits sur la peinture?

Précisément, fort peu de choses. On aurait pu croire que la nouvelle manière de penser le beau conduirait à un certain formalisme, et ce pour deux raisons. Pour une raison positive d'abord: parce que le beau nouvellement pensé est décrit en termes de propriétés formelles. Pour une raison négative ensuite: parce que la fin de la conception métaphysique du beau signe la fin de la convertibilité des transcendantaux et qu'elle invite à cesser de confondre le beau avec le bien ou avec le vrai. Or, il n'en est rien: Diderot défend une théorie humaniste des beaux-arts et pratique une critique éthique de la peinture.

L'expérience de l'art n'est pas pour Diderot une expérience étroitement aisthétique. La peinture s'adresse à l'esprit et non à la sensibilité. Elle est très 
précisément «l'art d'aller à l'âme par l'entremise des yeux» ${ }^{37}$. Condamnant par anticipation toute approche rétinienne de cet art, Diderot déclare: «je me soucie bien que l'artiste ait disposé ses figures pour les effets les plus piquants de lumières, si l'ensemble ne s'adresse point à mon âme ${ }^{38}$. Le peintre qui ne voudrait susciter que des expériences strictement esthétiques ne produirait que des œuvres inaccomplies: «si l'effet s'arrête aux yeux, le peintre n'a fait que la moindre partie du chemin ${ }^{39}$.

$S^{\prime}$ " arrêter aux yeux», c'est faire de l'art une expérience sensorielle et négliger ses possibles effets affectifs, cognitifs et comportementaux. C'est sacrifier le docere et le movere au placere. Or, l'Art poétique d'Horace, référence capitale pour l'Antiquité classique, le Moyen Âge et la Renaissance, liait ces trois finalités dans ce passage fameux et indéfiniment glosé: «les poètes entendent soit être utiles, soit faire plaisir, soit écrire des poèmes à la fois utiles et agréables à la vie», donc "tous les suffrages reviennent à celui qui a mêlé l'utile à l'agréable, en donnant au lecteur du plaisir et de l'instruction ${ }^{40}$. À la faveur du rapprochement opéré par la doctrine de l'Ut pictura poesis qui voit le jour à la Renaissance, la peinture doit, elle aussi, viser à instruire. En soutenant que l'art en général et la peinture en particulier ont une destination morale, Diderot se rallie donc à une tradition ancienne et toujours très prégnante chez ses contemporains.

L'art, affirme-t-il, a une fonction éthique: il doit «éterniser les grandes et les belles actions, [...] honorer la vertu malheureuse et flétrie, flétrir le vice heureux et honoré» ${ }^{41}$. Les artistes ont un devoir moral: "Rendre la vertu aimable, le vice odieux, le ridicule saillant, voilà le projet de tout honnête homme qui prend la plume, le pinceau ou le ciseau ${ }^{42}$. Ces derniers sont d'ailleurs comptés parmi «les précepteurs du genre humain, les consolateurs des maux de la vie, les vengeurs du crime, les rémunérateurs de la vertu ${ }^{43}$. À propos de La Piété filiale de Greuze, Diderot écrit dans son Salon de 1763: «Ne devons-nous pas être touchés de voir [le pinceau] concourir enfin avec la poésie dramatique à nous toucher, à nous instruire, à nous corriger, et à nous inviter à la vertu? Courage, mon ami Greuze! Fais de la morale en peinture, et fais-en toujours comme cela ${ }^{44}$. Trois ans plus tard, dans

37. D. Diderot, Salons, II. Salon de 1765, E. M. Bukdahl et A. Lorenceau (éd.), Paris, Hermann, 1984 , p. 226.

38. D. Diderot, Essais sur la peinture, p. 61.

39. D. Diderot, Salons, II. Salon de 1765, p. 226.

40. Horace, Art poétique, vers 333-334 et 344-345. Nous traduisons et soulignons.

41. D. Diderot, Essais sur la peinture, p. 61.

42. Ibid., p. 60.

43. Ibid., p. 61.

44. D. Diderot, Salon de 1763, in Essais sur la peinture - Salons de 1759, 1761, 1763, p. 234. 
De la poésie dramatique, il écrit encore: «Ô quel bien il en reviendrait aux hommes si tous les arts d'imitation se proposaient un objet commun et concouraient un jour avec les lois pour nous faire aimer la vertu et haïr le vice! C'est au philosophe à les y inviter; c'est à lui à s'adresser au poète, au peintre et au musicien et à leur crier avec force: Hommes de génie, pourquoi le ciel vous a-t-il doués?» ${ }^{45}$.

Une telle visée commande un certain nombre de recommandations poiétiques. La moralisation suppose des histoires édifiantes. Il faut que la peinture soit narrative, expressive, pathétique, vraisemblable. La forme ne doit pas opacifier la relation au référent. L'illusionnisme de la représentation est nécessaire pour que se produise l'immersion fictionnelle, qui elle-même est condition de la réaction affective et, in fine de l'effet éthique recherché. Par cet ensemble cohérent de prescriptions, Diderot se rattache à une poḯtique humaniste de la peinture.

En tant que critique, Diderot juge ainsi des œuvres en fonction de critères esthétiques mais aussi de critères extra-esthétiques. Il prend en considération les contenus éthiques des tableaux dans leur évaluation, comptant un défaut éthique comme un défaut artistique et une qualité éthique comme une valeur artistique. Le blâme adressé à Boucher et les louanges prodiguées à Greuze procèdent de là. Le premier, en peignant l'immoralité, la débauche ou la dépravation «pervertit le but des arts» ${ }^{46}$. Le second se conforme à ce que "tout morceau de sculpture ou de peinture doit être", à savoir «l'expression d'une grande maxime, une leçon pour le spectateur $"{ }^{47}$. Diderot fait donc du critique le représentant d'un ordre global à l'intérieur duquel valeurs esthétiques et valeurs éthiques sont étroitement liées.

Certes, d'autres passages des Salons sont infidèles à cette doctrine humaniste. Ce sont ceux dans lesquels se trouve affirmée la supériorité de la représentation sur le représenté. C'est notamment le cas dans le fameux commentaire de La Raie de Chardin, où l'attention se déplace du représenté vers la représentation, de l'objet peint à la manière dont il est peint. Le dégoût se mue alors en admiration. L'excellence de l'exécution retient le regard et le rend intransitif. C'est dire que la peinture est susceptible de produire une jouissance spécifique qui tient à ses qualités picturales propres. Ces passages laissent entrevoir la possibilité d'un plaisir proprement esthétique, lié à la picturalité du peint et rendu possible par

45. D. Diderot, De la poésie dramatique, in Entretiens sur "Le Fils naturel" - De la poésie dramatique - Paradoxe sur le comédien, J. Goldzink (éd.), Paris, Flammarion, 2005, p. 172.

46. D. Diderot, Essais sur la peinture, p. 60.

47. D. Diderot, De la poésie dramatique, in CEuvres esthétiques, p. 765. 
une attention intransitive. Mais une telle approche, si elle accorde à la forme la capacité à produire une jouissance particulière et légitime, ne fait pas de cette dernière une fin en soi. Ainsi, dans son commentaire de L'Accordée de village de Greuze, Diderot loue la composition pyramidale du tableau: «il y a douze figures, chacune est à sa place et fait ce qu'elle doit. Comme elles s'enchaînent toutes! Comme elles vont en ondoyant et en pyramidant!»; mais il ajoute aussitôt: «je me moque de ces conditions; cependant quand elles se rencontrent dans un morceau de peinture par hasard, sans que le peintre ait eu la pensée de les y introduire, sans qu'il leur ait rien sacrifié, elles me plaisent ${ }^{48}$. Le souci de la forme ne doit pas être le premier souci du peintre; les émotions qu'elle provoque sont légitimes mais subsidiaires. Les formes sont avant tout au service des contenus.

L'écart entre une approche naturaliste du beau et une approche humaniste de l'art ne se constate pas que dans l'œuvre de Diderot. Sous des formes diverses, on le trouve aussi chez d'autres auteurs du siècle: chez Hutcheson, chez Kant, ou encore chez Hogarth. Considérons un instant le cas de ce dernier, qui partage avec Diderot la double qualité d'être à la fois un théoricien et un artiste. Hogarth publie en effet en 1753 une Analyse de la beauté qui vise à répondre à cette seule question, qui est aussi celle de Hutcheson et de Diderot: en quoi consiste la beauté dans l'objet? «[J]e tâcherai, écrit-il dans l'introduction de l'ouvrage, de démontrer quels sont les principes de la nature d'après lesquels nous sommes déterminés à regarder certaines formes comme belles et gracieuses, et d'autres comme laides et désagréables » ${ }^{49}$. Ces principes sont au nombre de six: «la convenance, la variété, l'uniformité, la simplicité, la complication et la quantité, lesquelles contribuent toutes à produire en toute composition la beauté, en se corrigeant ou en se fortifiant mutuellement selon qu'il en est besoin ${ }^{50}$. Aussi la beauté du groupe sculpté antique du Laocoon ne tient pas à la grandeur de son sujet ou à la noblesse de l'attitude du prêtre Laocoon; elle réside dans la forme pyramidale de l'ensemble: "les trois statuaires qui ont exécuté le magnifique groupe du Laocoon, sans équivalent tant ancien que moderne, ont préféré tomber dans une faute grave, en donnant aux deux fils, quoique représentés d'ailleurs comme adultes, seulement la moitié de la grandeur de leur père, afin que par ce moyen l'ensemble de leur composition prît une forme pyramidale ${ }^{51}$. Mais il semble ne rester que peu de choses de ce souci de beauté formelle

48. D. Diderot, Salon de 1761, in Essais sur la peinture - Salons de 1759, 1761, 1763, p. 164-165.

49. W. Hogarth, Analyse de la beauté: destinée à fixer les idées vagues qu'on a du goût [1753], H. Jansen et S. Chauvin (éd.), Paris, École nationale supérieure des beaux-arts, p. 45.

50. Ibid., p. 54 (nous soulignons).

51. Ibid., p. 63. 
dans l'œuvre picturale ou gravée du peintre, qui inventa le genre des sujets moraux modernes. Ainsi Hogarth peint-il, en six tableaux, l'histoire d'une paysanne sombrant dans la prostitution et parvenant à en sortir (A Harlot's Progress, 1731), ou le destin tragique d'un débauché (A Rake's Progress, 1735). Ce sont là des œuvres lisibles et même narratives, qui appellent un regard transitif, sollicitent des réactions morales et visent des effets extra-esthétiques. Comme Diderot dans ses Salons, Hogarth est donc contenuiste dans sa pratique picturale; comme Diderot dans ses Recherches, il est formaliste dans son analyse théorique.

Ainsi donc, les changements radicaux qui affectent la manière d'entendre la beauté ne produisent pas d'effets immédiats dans le champ des arts et des discours sur l'art. La callistique de Diderot relève d'une esthétique naturaliste, mais il ne réduit pas à celle-ci la beauté artistique, l'héritage humaniste constituant un contrepoids puissant à une telle "aisthétisation» de l'art. Conformément à cette tradition, il soutient une conception "contenuiste», transitive, référentielle de l'art, qui ne dissocie pas la forme de son contenu. Sans doute est-ce par cette conclusion qu'on peut apporter une réponse à la question posée plus haut: si Diderot continue à utiliser des formules liant le beau au bien et au vrai, des formules qui donc renvoient à une vision du beau qu'il récuse sur le plan théorique, c'est généralement dans des contextes où il est question d'art. C'est dans les Essais sur la peinture qu'il écrit que «Le vrai, le bon et le beau se tiennent de bien près. Ajoutez à l'une des deux premières qualités quelque circonstance rare, éclatante, et le vrai sera beau, et le bon sera beau ${ }^{52}$. C'est dans Le Neveu de Rameau qu'il pose entre eux un lien d'engendrement ${ }^{53}$. Tout se passe donc comme si - dans l'œuvre de Diderot et au-delà d'elle - le champ artistique n'avait pas été atteint (pas encore atteint faudrait-il dire) par les conséquences de la révolution épistémique touchant le beau.

Carole TAlON-Hugon

Université de Nice - Sophia Antipolis

52. D. Diderot, Essais sur la peinture, p. 76.

53. D. Diderot, Le Neveu de Rameau, p. 462. 